\title{
Primary intracranial fibrosarcoma: Review of management options with cases illustration
}

\author{
Satyarthee GD* \\ Department of Neurosurgery, Room no. 714, Neurosciences Centre, AIIMS New Delhi, India
}

\begin{abstract}
Introduction: Primary intracranial fibrosarcoma without prior irradiation is very rare occurence. We compare the effect of different modalities of treatment of primary intracranial fibrosarcoma.

Case-report: A 30 -years- old male underwent a complete excision of right frontoparietal mass, Histopathological diagnosis was fibrosarcoma. He received chemotherapy and irradiation. He was doing well at 18 months following primary cranial surgery.
\end{abstract}

Conclusion: Early diagnosis and prompt surgical excision followed by chemotherapy and radiotherapy can be started early after the surgical excision.

\section{Introduction}

Primary intracranial fibrosarcoma originating within the brain parenchyma or its meningeal cover are uncommon; it accounts for $1.5-2 \%$ of all intracranial neoplasm [1,2]. These tumours are thought to originate from mesenchymal cells in dura mater, leptomeninges, vascular adventitia, or stalk or choroid plexus [3,4]. Ultra-structural studies have suggested their origin from fibroblast

The meningothelial origin of fibrosarcoma-appear to be one of the most common histology including malignant" fibrous histiocytoma $[5,6]$. Tumour mass is usually located in the supratentorial compartment and often located superficially and adherent to dura mater. We report a case of fibrosarcoma located in the right frontoparietal region.

\section{Case illustration}

A 34 -year old male presented with right focal motor seizure with secondary generalization for one year, which was not controlled with combination antiepileptic medication including phenytoin and phenobarbitone in adequate dosages, $\mathrm{He}$ had postictal right Sided hemiparesis lasting for few weeks. On admission history included full course of antitubercular therapy for pulmonary Koch's two years back. Neurological examination revealed bilateral papilloedema. Right sided upper motor neuron seventh cranial nerve paresis. Other cranial nerves were normal. Motor examination revealed hypertonia involving right upper and lower limb with muscle power of four (MRC). Right plantar reflex was extensor. Sensory examination revealed loss of cortical sensation over right side the body. Cranial computed tomography scan revealed $4.5 \mathrm{X} 4 \mathrm{~cm}$ in size and homogeneous, well-circumscribed mass located in the left parietal region with extension into posterior frontal lobe and marked perilesional edema and causing subfalcine herniation.

The patient underwent left pericoronal osteoplastic craniotomy with Midas drill. After dural opening, a white's greyish mass of hard consistency was visible on the surface. The tumour was nonencapsulated, and complete surgical excision was carried out. The histological diagnosis was fibro sarcoma. Histopathology showed a highly cellular tumour, consisted of sheets of spindle shaped cells in fascicular, arrangement, nuclear pleomorphism and high mitotic activity was observed. However, areas of necrosis were not evident. Immunohistochemical study demonstrated positivity for vimentin but negative for glial fibrillary acidic protein. The further systemic workup of the whole body including a x-Jay chest, CT scan chest, and abdomen, ultrasound abdomen showed no extracranial disease foci could be located. The spinal $\mathrm{x}$-ray also was normal. He was referred to chemotherapy and received vincristine, isosfamide and Adriamycin in combination with steroid were administered. A postoperative CT scan was normal; additionally also received a total of 56 Gy radiation therapies were given over 28 fractions. After surgery, although the seizure frequency has markedly reduced but mild hemiparesis still persisting. At the last follow-up at three years he was disease free

\section{Discussion}

Primary fibrosarcoma of the brain or meninges are very rare intracranial tumour, and its incidence in the previously reported series was $0.5-2 \%[1,2]$. However, more recent series suggested a very low incidence of 1 case, -among 402 children reported by Tomita and Gonzales - Cruse [6]. Further Paulus et al. reported only one case among 25,000 brain tumours. Thus, the changing incidence may be attributed to improvement in the diagnostic tools and changes in the classification [5]. These tumours are not associated with any typical symptomatology or signs that would be helpful in differentiating it from other central nervous system neoplasm $[7,8]$.

Our patient had seizure as initial presentation several mechanisms are postulated for seizure associated with tumour [9]. Metabolic

${ }^{\star}$ Correspondence to: Guru Dutta Satyarthee MCh, Associate Professor, Department of Neurosurgery, Room no. 714, Neurosciences Centre, AIIMS New Delhi, India, E-mail: duttaguru2002@yahoo.com

Key words: primary intracranial fibrosarcoma, surgery, management

Received: January 03, 2019; Accepted: January 10, 2019; Published: January 16, 2019 
disturbance, $\mathrm{pH}$ abnormalities, amino acid and neuro-receptor disturbance and immunological activity. The most plausible mechanism is intrinsic property of tumour cells membrane and their ability to generate action potential, $\mathrm{pH}$ disturbances, which directly caused alteration in neuronal excitability, possible through secondary effect on NMDA subclass of glutamate receptor. The focal seizure, which represents the brain hypo-function, with a variety of feature.

Radiation induced fibrosarcoma usually show a very long latent period of two years to 24 years but can occur even within several months following radiotherapy [9]. Gaspar et al. [2] reviewed nine cases of primary cerebral fibrosarcoma, four arose deep within cerebral hemisphere and remaining five cases involved the leptomeninges at cortical surface, these tumours may have very high rate of local recurrence. The recurrence usually occurred over a period ranging from few months to 5 years7 years but commonly within one year of surgery [8]. However, patients may present with chronic subdural haematoma due to meningeal spread.

In our case the diagnosis of the primary intracranial fibrosarcoma was based on deatailed systemic survey, which did not reveal any other foci and histopathology confirmed the diagnosis of fibrosarcoma. The spindle cells in fascicular pattern the tumour with high mitotic rate and high cellular tumour. However, the sarcomata's transformation of the pre-existing brain neoplasm i.e. glioma and meningioma were excluded by negative Immunostaining of glial fibrillary acidic protein and epithelial membrane antigen respectively. Bisogno et al in a detailed literature search could get only 20 case report of primary intracranial fibrosarcoma and concluded very uncommon occurrence in paediatric population $[2,5,6]$.

The management is still controversial but surgical excision followed by radiotherapy and chemotherapy advocated. Gaspar et al observed high incidence ( $44 \%$ ) of meningeal seeding and $44 \%$ had systemic metastasis. Documented spinal arachnoid spread in two out of eight cases. These tumours presents with aggressive behaviour and used to invariably recur at the primary site despite aggressive surgical resection. Time for recurrence progression remains highly variable. It has local tendency to recur as fibrosarcoma doing elsewhere in the human body.

Extended surgical resection of intracranial sarcoma is not possible unlike sarcoma involving limbs Wide en block surgical excision cannot be performed $[10,11]$. Radiation therapy of 64-66 Gy is required for residual extremity sarcoma [10] but these represent the maximal tolerable limit of the normal brain. Bisogno et al 8 advised radiation therapy for residual tumour. however, they did not recommenced craniospinal irradiation. Chang et al "disputed the role of adjuvant chemotherapy in treatment of fibrosarcoma irrespective of its location. However, Bisogno et reported good outcome with virtual disappearance of tumour and patient doing well were further advised radiotherapy and chemotherapy 44 month after the treatment. Overall, the prognosis of intracranial fibrosarcoma is poor. In 1984. Tomita and Gonzales reviewed 18 children with primary Central nervous system fibrosarcoma, only five were alive after 8 months only It is imperative that a combination of good surgical resection followed by early chemotherapy and radiotherapy can help in prolonging the recurrence free survival at least few months. It can be a good palliative therapy. In our case also chemotherapy and radiotherapy were administered after three months delay following the wide surgical excision.

\section{Summary}

The prognosis of intracranial fibrosarcoma is poor. As wide excision of intracranial fibrosarcoma is not possible, adequate microsurgical excision, however, priority based the adjuvant chemotherapy and radiotherapy should be administered with a hope to prolong the recurrent free survival. However, being a rare entity. Ideal management modalities require a study involving larger size cohort in future.

\section{References}

1. Christensen E, Lara DE (1953) Intracranial sarcomas. J Neuropathol Exp Neurol 12 41-56. [Crossref]

2. Caspar LE, Mackenzie IRA, Gilbert JJ, Kauffmann JCE, Fisher BF, et al. (1993) Primary cerebral fibrosarcoma's- clinicopathologic study and review of literature. Cancer 72: 3277- 3281.

3. Nichols P Jr, Wagner JA (1952) Primary intracranial sarcoma; report of nine cases with suggested classification. J Neuropathol Exp Neurol 11: 215-234. [Crossref]

4. Russel DS, Rubenstein LJ (1989) Pathology of the tumours of the nervous system. 5 th ed.Baltimore: Williams and Wilkins 1989 .

5. Paulus W, Slowik F, Je1linger K (1991) Primary intracranial sarcomas: histological features of 19 cases. Histopathology 18: 395- 402.

6. Tomita T, Gonzales - Crussi F. intracranial primary non-lymphomatous sarcomas in the children: experience with eight cases and review of literature. Neurosurg 99984: 14: 529-540.

7. Mena H, Garcia ill (1978) Primary brain sarcomas, light and electron microscopic features. Cancer 42: 1298- 1307.

8. Bisogno G, Roganovic J, Carli M, Scarzello G, Calderone M, et al. (2002) Primary intracranial fibrosarcoma Childs Nerv Sys 8: 648-651.

9. Beaumont A, Whittle IR (2000) The pathogenesis of tumour associated epilepsy. Acta Neurochir (Wien) 142: 1-15. [Crossref]

10. Giuliano AE, Eilber FR (1985) The rationale for planned reoperation after unplanned total excision of soft tissue sarcoma. J Clin Oncol 3: 1344-1348.

11. Chang AE, Kinsella T, Glatstein E, Baker AR, Sindelar WF, et al. (1988) Adjuvant chemotherapy for patients with high-grade soft-tissue sarcomas of the extremity. J Clin Oncol 6: 1491-1500. [Crossref]

Copyright: $@ 2019$ Satyarthee GD. This is an open-access article distributed under the terms of the Creative Commons Attribution License, which permits unrestricted use, distribution, and reproduction in any medium, provided the original author and source are credited. 